\title{
Charnley low-friction arthroplasty of the hip. Five to 25 years survivorship in a general hospital Daniel Hernández-Vaquero*1,2, Abelardo Suárez-Vazquez ${ }^{1}$ and Jesus Fernandez-Lombardia ${ }^{1}$
}

Address: ${ }^{1}$ Department of Orthopaedic Surgery, Hospital St Agustin, Aviles, Spain and ${ }^{2}$ School of Medicine, University of Oviedo, Spain Email: Daniel Hernández-Vaquero* - dhernandezv@meditex.es; Abelardo Suárez-Vazquez - asuarezv@telecable.es; Jesus FernandezLombardia - jesusflo@telecable.es

* Corresponding author

Published: 15 May 2008

BMC Musculoskeletal Disorders 2008, 9:69 doi:10.1186/1471-2474-9-69

This article is available from: http://www.biomedcentral.com/l47/-2474/9/69

(c) 2008 Hernández-Vaquero et al; licensee BioMed Central Ltd.

This is an Open Access article distributed under the terms of the Creative Commons Attribution License (http://creativecommons.org/licenses/by/2.0), which permits unrestricted use, distribution, and reproduction in any medium, provided the original work is properly cited.

\begin{abstract}
Background: Some studies have raised the question about whether the good results obtained with the Charnley prosthesis could be replicated at general hospitals when it comes to the frequency of early complications and failure rates, both of which would be higher than those published by centres devoted to hip arthroplasties.
\end{abstract}

Methods: We reviewed the results of 404 Low Friction Arthroplasties of the hip implanted between 1976 and 1993 in a general hospital by general orthopaedic surgeons. For the survival analysis, the end-point chosen would be the chirurgical revision of any of the prosthetic components for whatever reason.

Results: The complications were 16 dislocations (4\%), 14 deep infections (3.5\%), 2 neurological injuries $(0,5 \%)$ and 5 clinical deep venous thromboses (I.2\%) (2 pulmonary embolisms). The survival rate at 25 years, both for stem and cup, was $83 \%$. Survival was higher in those arthroplasties implanted in patients older than 60 years, with statistical significance.

Conclusion: Low Friction Arthroplasty undertaken at general hospitals by general orthopaedic surgeons feature similar outcomes to those found in centres devoted to hip surgery.

\section{Background}

Low Friction Arthroplasty (LFA) is still considered the "golden guide" when it comes to compare the different Total Hip Arthroplasy (THA) models. Many studies have been published on LFA, showing excellent, long-term results, both about clinical and radiological issues $[1,2]$ as well as about survival analyses [3-5]. Nevertheless, most of these studies include series of LFA undertaken at hospitals which were pioneers in this technique [6], or which were devoted to hip surgery procedures. Some studies have raised the question about whether the good results obtained with the Charnley prosthesis could be replicated at general hospitals when it comes to the frequency of early complications and failure rates, both of which would be higher than those published [7]. Survival analysis is a powerful tool for analysing the results of total joint replacements despite the objections found in the literature [8], but it has major drawbacks when the failure rates are very low.

Our Unit belongs to a general hospital located in northern Spain, and it serves a population of 180,000; it is not spe- 
cifically devoted to hip surgery. Since its opening in 1976, the hospital has remained faithful to LFA throughout the years (Figure 1). Our aim is to know the long term behaviour of these arthroplasties by studying any eventual complications which may have arisen and by conducting a survival analysis.

\section{Methods}

404 LFA (294 patients) performed as primary procedures consecutively from 1976 to 1993 have been included in this study. In all cases the implant used was the prosthesis designed by J. Charnley, with the technical and design improvements added throughout the years [9]. The surgical procedures were undertaken by a team of six general orthopaedic surgeons following a homogeneous technique, always using a Smith-Petersen anterior approach without performing the osteotomy of the greater trochanter. The senior surgeon (DHV) had previously attended to the Center for Hip Surgery at Wrightington,

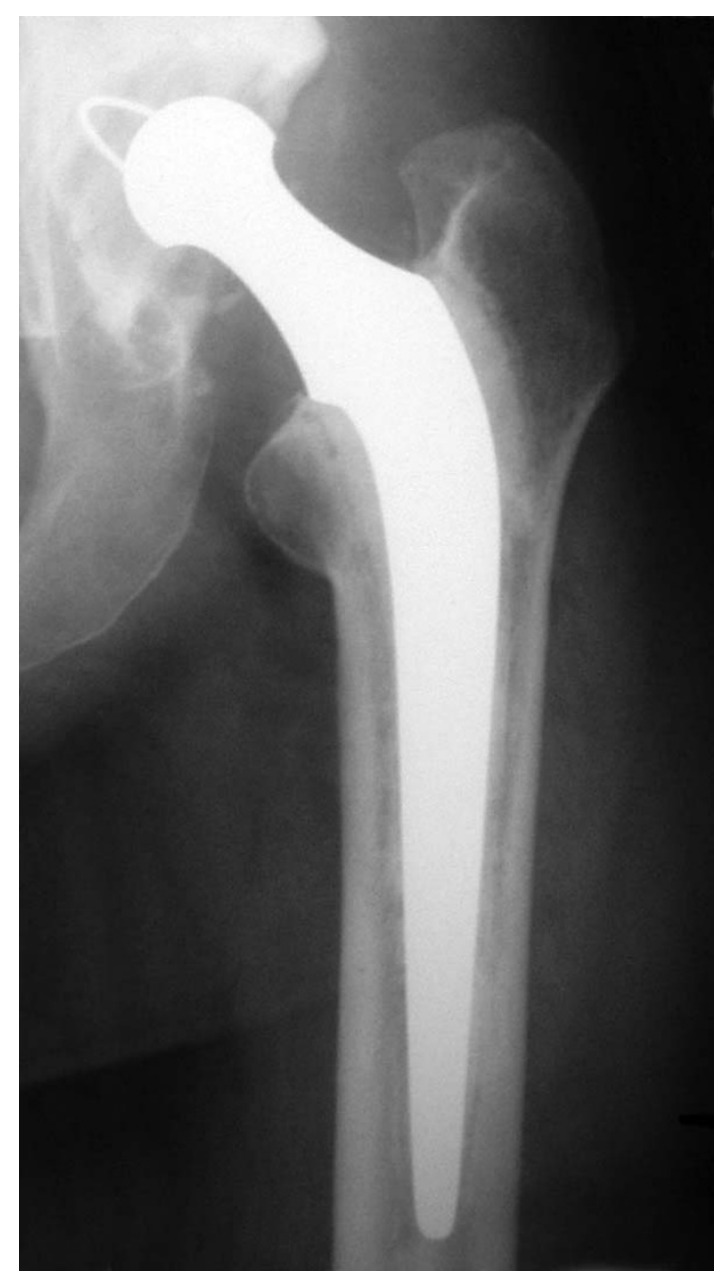

Figure I

Low friction arthroplasty with 22 years follow-up. No complications.
England in 1976 and 1979 to learn the original technique. No mechanical cementing system was used.

Throughout 2004, two independent observers who did not participate in the surgery (ASV and JFL) reviewed the medical histories and radiographies of each patient, categorizing the radiographs into those that were possibly, probably or definitely loose. General data about the patient and the intervention as well as about the clinical development were gathered. Since we are dealing with a long-term, retrospective study, only easily verifiable variables (age, sex, side) were accepted. Any complications which required an active medical intervention and which, accordingly, were featured in the medical histories, were also included.

For the survival analysis, the end-point chosen would be the chirurgical revision of any of the prosthetic components for whatever reason. Variable data (time, event, age and sex) were recorded in a SIGMASTAT v 3.00 for Windows (SPSS, Inc., Chicago, IL) database built for such purpose. The survival calculations were done with this tool, following the Kaplan-Meier method [10] with a confidence interval of $95 \%$. The equality of survival distributions was contrasted for the acetabulum and stem components through the Log Rank test. In every hypothesis test, only $\mathrm{p}<0.05$ values were considered statistically significant.

\section{Results}

The mean age at the moment of surgery was of 67 years (SD: 8.8), where the maximum age was 91 years and the minimum was $36.57 \%$ of the cases were males and $43 \%$ females; $45 \%$ were right side and 55\%, left side.

The following complications were registered: 16 dislocations (4\%), 14 deep infections (3.5\%), 5 periprosthetic fractures $(1.2 \%), 2$ neurological injuries (in the crural nerve and 1 in the sciatic nerve), 5 deep venous thromboses $(1.2 \%)$ with 2 pulmonary embolisms, one of them fatal.

After 25 years, the cup and stem survival rates were of $83 \%$ (Table 1 and 2), (Figure 2). A 55.2\% of arthroplasties were lost by dead of patients and $20 \%$ by unknown causes [see

Table I: Survival rate of the cup

\begin{tabular}{lcc}
\hline Follow-up (years) & LFA & Cup survival (\%) \\
\hline 5 & 307 & 96 \\
10 & 235 & 93 \\
15 & 128 & 91 \\
20 & 57 & 89 \\
25 & 10 & 83
\end{tabular}


Table 2: Survival rate of the stem

\begin{tabular}{lcc}
\hline Follow-up (years) & LFA & Stem survival (\%) \\
\hline 5 & 305 & 95 \\
10 & 230 & 92 \\
15 & 123 & 87 \\
20 & 52 & 83 \\
25 & 10 & 83 \\
\hline
\end{tabular}

Additional file 1]. The acetabular component demonstrated a better behaviour at the beginning of the followup, but at the end it equals that of the femoral stem (though it has no statistical significance). Figure 3 shows the survival curve of the components after grouping the series according to the age of the patients (younger and older than 60 years). Survival was higher in those arthroplasties implanted in patients older than 60 years, with statistical significance in the Log Rank test, both for the acetabular component $(p=0.008)$ and the femoral stem component $(p=0.043)$, with a higher difference between the curves as development time increases. There were no differences related to sex.

\section{Discussion}

A surgical technique is reproducible if its results are similar in real, non-ideal, conditions, and that is what seems to happen with LFA. In the long term, the survival rate of

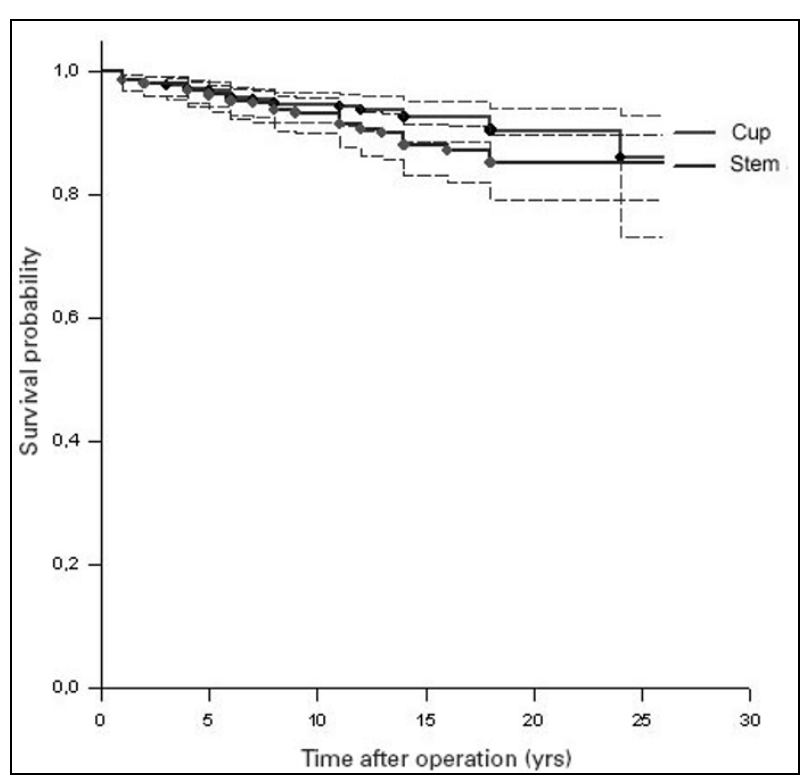

Figure 2

Kaplan-Meier survival curve showing the cumulative survival per year.

LFA in our centre is very similar to those shown in historical series or arthroplasty register $[11,12]$ and in those centres devoted to hip surgery $[3,4,13]$ The worst survival rate

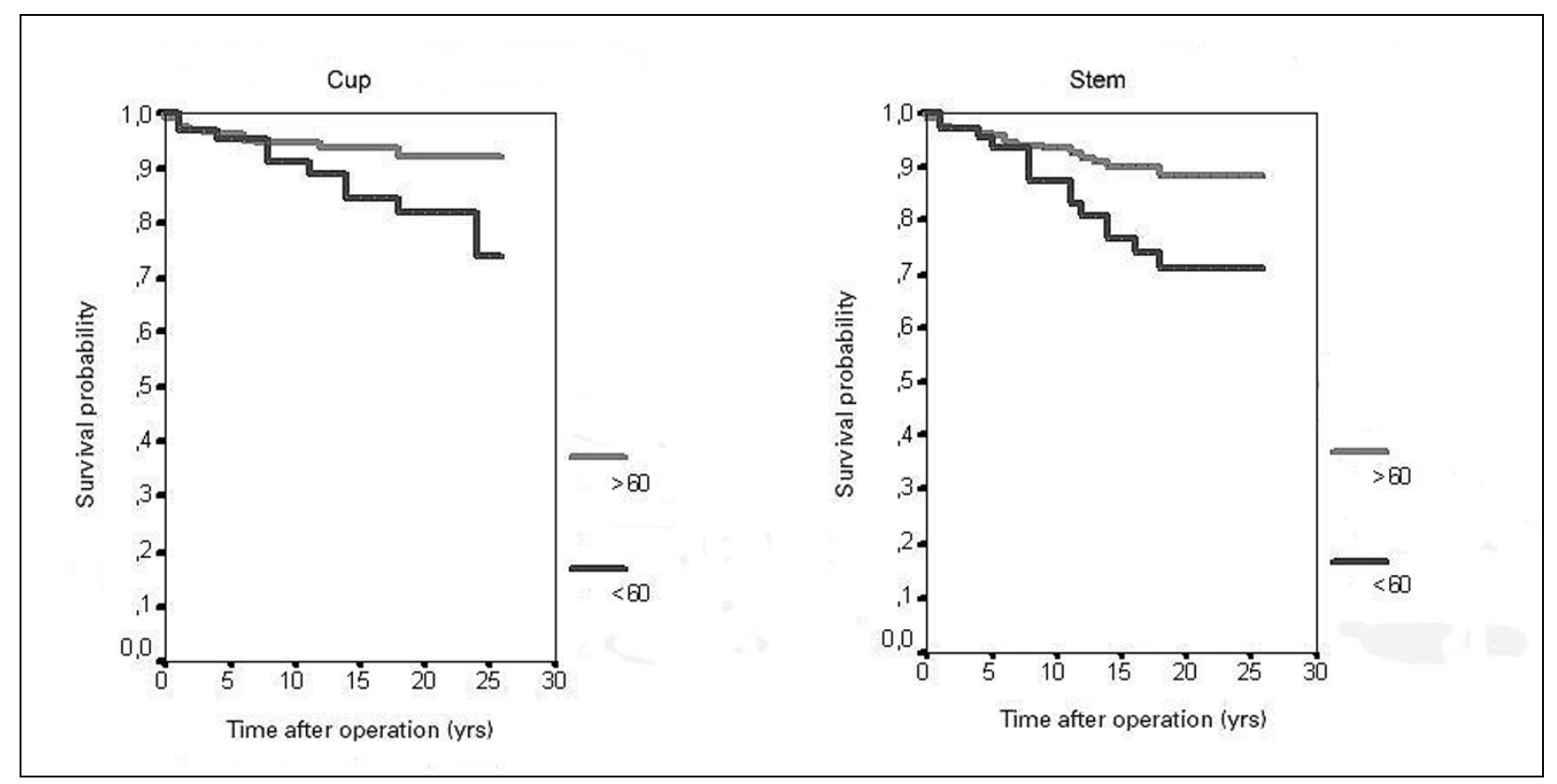

Figure 3

Survival curve of the components after grouping the series according to the age of the patients. 
is found in the youngest patients, just as these authors proved [12]. In the short-term, however, our survival rate is worse than those found in the aforementioned series, being closer to the results expressed by Fender et al [7]. Our deep infection rate is high, since it includes procedures undertaken in a time when antibiotic prophylaxis was not systematically used and this may justify, if only partially, such results.

Both in our series as well as in a multicentric series [14], the global survival rate was of $83 \%$ after 20 years. There are, however, some minor differences when we analyze the survival of each component on its own, around $87 \%$ for each of them in Older's multicentric series, and 83\% and $89 \%$ for the acetabular and stems, respectively, in our series.

The dislocation rate is also higher than the classical rate of $2 \%-3 \%$ for primary surgery [15] being closer to $3.9 \%$, which can be found in those studies undertaken at general facilities [16]. However, it is still inferior to that published by Fender et al which reached 5\%.

The assessment of results in THA requires a multiple approach, either through clinical score sets complemented with imaging analyses, curves or survival analysis, or through score and review indices about the quality of life related to health. Each approach addresses a requirement of the complex evaluation of this technique which, paradoxically enough, must be reviewed in the long term in spite of being relatively new and rapidly evolving in developed countries.

There remain many unresolved questions in hip replacement. Well-constructed clinical tests offer the best hopes of answering them. Such tests are onerous, and time and money are required to collect, record and analyze the data. The relatively few clinicians who undertake such tests must have an understanding of the statistical methods used and the problems they may encounter. It is especially difficult to find series where you can compare long term results in the literature and whether the arthroplasty was implanted in general hospitals. Only retrospective studies are available, which must be accepted in spite of the known issues with validity. The methodological flaws common to these studies could be alleviated using, as in our case, data about which there is absolute certainty they are true, even though this would mean an obvious loss of information. In joint replacement surgery the usual endpoint is the decision to remove the prosthesis and revise or convert it to some other form of treatment. This endpoint has been criticized because the criteria used to decide the need for removal will differ between patients and surgeons. This form of analysis defines a failure point or terminal event and provides an assessment of not only how many failures there had been but also how long after the operation did the failure occur. It does not measure function or pain, unless they are included in the definition of failure (Figure 4). Using a confidence interval of $95 \%$ in the survival curve allows a more faithful comparison of the best and worst possible results with those of other series.

To sum up, our study seems to confirm a higher frequency of early complications and a higher assessment rate of the THA in general hospitals compared to those in dedicated centres, although in the long term this trend does not keep up and the survival rates become similar. LFA undertaken

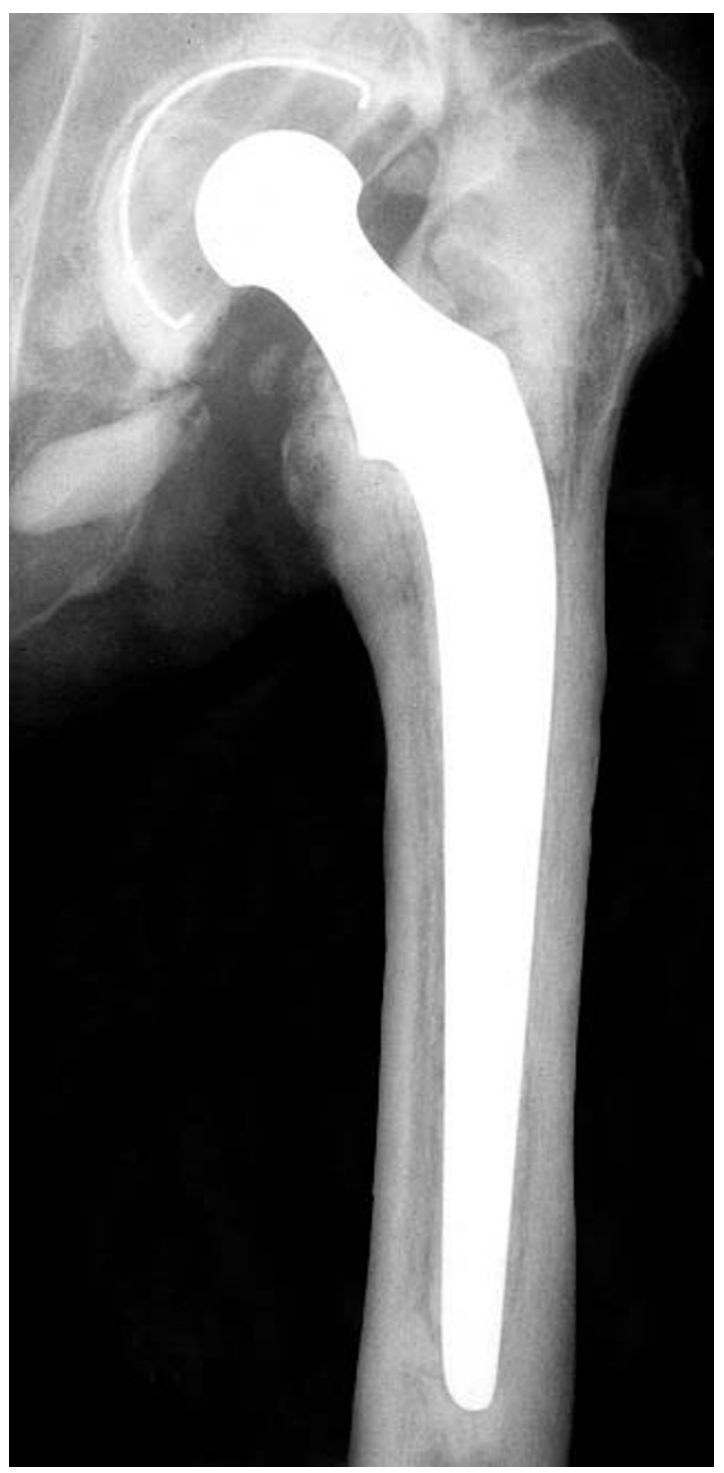

Figure 4

Stem loosening in a Charnley arthroplasty ( 17 years follow-up). 
at general hospitals by general orthopaedic surgeons feature similar outcomes to those found in centres devoted to hip surgery. A national joint replacement registry would be a useful tool to compare outcomes for high and low volume community hospitals as well as academic centres.

\section{Conclusion}

Low Friction Arthroplasty undertaken at general hospitals by general orthopaedic surgeons feature similar outcomes to those found in centres devoted to hip surgery.

\section{Abbreviations}

THA: Total hip arthroplasty; LFA: Low friction arthroplasty.

\section{Competing interests}

The authors declare that they have no competing interests.

\section{Authors' contributions}

DHV conceived of the study, participated in its design and coordination and drafted the manuscript, ASV and JFL reviewed the medical histories and radiographies. All authors read and approved the final manuscript.

\section{Additional material}

\section{Additional file 1}

Survival tables. 3 tables.

Click here for file

[http://www.biomedcentral.com/content/supplementary/1471-

2474-9-69-S1.doc]

\section{Acknowledgements}

Dr. Fernandez-Carreira (Clinical Research Unit, Hospital St Agustin, Aviles, Asturias, Spain) performed the statistical analysis.

\section{References}

I. García-Cimbrelo E, Diez-Vazquez V, Madero R, Munuera L: Progression of radiolucent lines adjacent to the acetabular component and factors influencing migration after Charnley lowfriction total hip arthroplasty. I Bone Joint Surg (Am) 1997, 79: $1373-1380$

2. Wroblewski BM, Fleming PA, Siney PD: Charnley low-frictional torque arthroplasty of the hip. 20- to $\mathbf{3 0}$ year results. J Bone Joint Surg $(\mathrm{Br})$ 1999, 8 I:427-430.

3. Callaghan JJ, Templeton JE, Liu SS, Pedersen DR, Goetz DD, Sullivan PM, Johnston RC: Results of Charnley total hip arthroplasty at a minimum of thirty years. A concise follow-up of a previous report. J Bone Joint Surg (Am) 2004, 86:690-695.

4. Berry DJ, Harmsen WS, Cabanela ME, Morrey BF: Twenty-fiveyear survivorship of two thousand consecutive primary Charnley total hip replacements: factors affecting survivorship of acetabular and femoral components. J Bone Joint Surg (Am) 2002, 84: 171-I77.

5. Devitt A, O'Sullivan T, Quinlan W: I 6-to 25-year follow-up study of cemented arthroplasty of the hip in patients aged 50 years or younger. J Arthroplasty 1997, I 2:479-489.

6. Wroblewski BM, Siney PD, Fleming PA: Charnley low-friction arthroplasty. Survival patterns to 38 years. J Bone Joint Surg $(B r)$ 2007, 89:1015-1018.
7. Fender D, Harper WM, Gregg PJ: Outcome of Charnley total hip replacement across a single health region in England. The results at five years from a regional hip register. J Bone Joint Surg $(\mathrm{Br})$ 1999, 81:577-581.

8. Murray DW, Carr AJ, Bulstrode C: Survival analysis of joint replacements. J Bone Joint Surg $(\mathrm{Br})$ 1993, 75:697-704.

9. Charnley J: Low Friction Arthroplasty of the Hip. In Theory and Practice Springer Verlag, Berlin-Heidelberg-New York; 198I.

10. Kaplan EL, Meier P: Nonparametric estimation from incomplete observations. J Am Stat Assoc 1958, 53:457-48I.

II. Allami MK, Fender D, Khaw FM, Shander DR, Esler C, Harper Wm, Gregg PJ: Outcome of Charnley total hip replacement across a single health region in England. The results at ten years from a regional arthroplasty register. J Bone Joint Surg (Br) 2006, 88: | 293- I 298.

12. Malchau H, Herberts P, Eisler T, Garellick G, Saderman P: The Swedish Total Hip Replacement Register. J Bone Joint Surg (Am) 2002, 84:2-20.

13. Wroblewski BM, Siney PD, Fleming PA: Charnley low-frictional arthroplasty in young rheumatoid and juvenile rheumatoid arthritis. Acta Orthop 2007, 78:206-210.

14. Older J: Charnley low-friction arthroplasty. A worldwide retrospective review at I5 to 20 years. J Arthroplasty 2002, I 7:675-680

15. Mahoney CR, Pellicci PM: Complications in primary total hip arthroplasty: avoidance and management of dislocations. Instr Course Lect 2003, 52:247-255.

16. Phillips CB, Barrett JA, Losina E, Mahomed NN, Lingard EA, Guadagnoli E, Baron JA, Harris WH, Poss R, Katz JN: Incidence Rates of Dislocation, Pulmonary Embolism, and Deep Infection During the First Six Months After Elective Total Hip Replacement. J Bone Joint Surg (Am) 2003, 85:20-26.

\section{Pre-publication history}

The pre-publication history for this paper can be accessed here:

http://www.biomedcentral.com/1471-2474/9/69/prepub
Publish with Bio Med Central and every scientist can read your work free of charge

"BioMed Central will be the most significant development for disseminating the results of biomedical research in our lifetime. "

Sir Paul Nurse, Cancer Research UK

Your research papers will be:

- available free of charge to the entire biomedical community

- peer reviewed and published immediately upon acceptance

- cited in PubMed and archived on PubMed Central

- yours - you keep the copyright

Submit your manuscript here:

http://www.biomedcentral.com/info/publishing_adv.asp
BioMedcentral 